\title{
The relationship between exercise capacity and neutrophil/ /lymphocyte ratio in patients taken to cardiopulmonary rehabilitation program
}

\author{
Okan S
}

Tokat State Hospital. Physical Medicine and Rehabilitation Tokat, Turkey. doctorsevil@yahoo.com

\begin{abstract}
OBJECTIVES: The aim of the present study was to evaluate the relationship between exercise capacity and n/lymphocyte ratio (NLR) in patients enrolled in a cardiopulmonary rehabilitation program.

BACKGROUND: NLR has recently been used as a potential marker to determine inflammation in cardiac and non-cardiac diseases.

METHODS: In this retrospective study, an exercise test and six-minute walking test (6MWT) were carried out in 23 patients with coronary artery disease and 28 patients with pulmonary disease before cardiopulmonary rehabilitation program, and routine hemogram test results were evaluated.

RESULTS: The result of 6MWT test distance was $333.43 \pm 86.58 \mathrm{~m}$ in the cardiac rehabilitation group and $348.46 \pm 81.37 \mathrm{~m}$ in the pulmonary rehabilitation group. There was a negative correlation between 6MWT and NLR in the cardiac rehabilitation group $(p<0.05)$. As NLR increased, the MET value and duration of exercise decreased in the pulmonary rehabilitation group $(p<0.05)$.

CONCLUSION: NLR could be used as a predictor to evaluate the exercise capacity in patients to be enrolled in cardiopulmonary rehabilitation program (Tab. 3, Ref. 30). Text in PDF www.elis.sk.

KEY WORDS: exercise capacity, neutrophil/lymphocyte ratio, rehabilitation
\end{abstract}

\section{Introduction}

Cardiovascular diseases and chronic obstructive pulmonary disease (COPD) are chronic conditions that could impair physical functionality, psychological health and life quality (1). Atherosclerotic cardiovascular diseases and chronic airway diseases (asthma and COPD) are among the ten most common diseases in Turkey (2). Inflammation plays a key role in the onset and progression of cardiovascular diseases, COPD and asthma. Neutrophil/lymphocyte ratio (NLR) has recently been used as a potential marker to determine inflammation in cardiac and non-cardiac diseases $(3,4)$. In addition, NLR has been found to be associated with disease severity, hospitalization, malnutrition, recurrence and mortality in various chronic conditions including cardiovascular diseases $(3,5)$. In a Japanese study dealing with the association between NLR value and clinical parameters in 141 individuals with COPD diagnosis, the elevated NLR values were associated with higher BODE index (body mass index, obstruction, dyspnea and exercise capacity). This finding means that NLR is associated not only with

State Hospital, Physical Medicine and Rehabilitation Tokat, Turkey

Address for correspondence: S. Okan, Physical Medicine and Rehabilitation, Tokat State Hospital Tokat, Turkey, 60100

Phone: +05308211797 airway obstruction but also with higher malnutrition, dyspnea and impaired exercise capacity (6).

Cardiopulmonary rehabilitation programs are considered a part of comprehensive care for the patients with cardiopulmonary disease (7). Maximum exercise capacity, which is among the core starting components of cardiac rehabilitation in all clinical conditions, is measured by symptom-limited exercise test (8). The most objective method to measure exercise capacity is cardiopulmonary exercise test which measures peak oxygen consumption (9). Nevertheless, cardiopulmonary exercise test cannot be used at any time and needs considerable level of expertise. Instead of using this test, the exercise capacity is generally measured indirectly and expressed as metabolic equivalent (MET) (10). Another method used to determine exercise capacity is the six-minute walking test (6MWT) (11). In a multicenter prospective observational study with 885 COPD patients, individuals with higher NLR levels were found to have shorter 6MWT distances (12).

The aim of the present study was to evaluate a possible association between NLR levels and exercise capacity in individuals planned to be enrolled in cardiopulmonary rehabilitation program.

\section{Methods}

This retrospective study included the starting data of 51 individuals who had atherosclerotic cardiovascular disease, COPD 
and asthma diagnoses and who were treated at the Cardiovascular Rehabilitation Unit in 2017-2018 period. Symptom-limited treadmill test and 6MWT test were performed for all patients based on symptom-limited treadmill test results and guidelines (13). Patients were asked to walk the longest distance in a marked area for six minutes. They were told that they could slow down or accelerate at their will. The test was terminated in patients who developed serious symptoms during the test. MET values were obtained from symptom-limited exercise test performed based on the Bruce protocol or its modified version (14). Hemogram test results were obtained from hospital information system. The neutrophil/lymphocyte ratio was calculated by dividing absolute neutrophil count by absolute lymphocyte count, while platelet/ lymphocyte ratio was obtained from dividing absolute platelet count by absolute lymphocyte count. Five patients whose hemogram test results could not be obtained and six patients whose symptom-limited exercise tests were carried out using bicycle ergometry were excluded. The study protocol was approved by the local ethic committee (19-KAEK-058).

\section{Statistical analyses}

The descriptive analyses were conducted to obtain information about general characteristics of the study groups. The data of

Tab. 1. Quantitative variables of cardiac and pulmonary rehabilitation groups.

\begin{tabular}{lccc}
\hline & \multicolumn{2}{c}{ Group } & \\
\cline { 2 - 3 } & $\begin{array}{c}\text { Cardiac } \\
\text { rehabilitation }\end{array}$ & $\begin{array}{c}\text { Pulmonary } \\
\text { rehabilitation }\end{array}$ & $\mathrm{p}$ \\
\cline { 2 - 3 } & Mean \pm SD & Mean \pm SD & \\
\hline Age & $60.04 \pm 6.79$ & $54.07 \pm 11.08$ & 0.241 \\
Resting peak heart rate & $83.00 \pm 13.91$ & $87.68 \pm 14.09$ & 0.133 \\
Exercise test peak heart rate & $142.52 \pm 15.27$ & $133.29 \pm 25.45$ & 0.067 \\
Exercise test peak heart rate (\%) & $87.48 \pm 9.29$ & $82.14 \pm 10.76$ & 0.146 \\
Borg score & $11.96 \pm 1.02$ & $12.39 \pm 1.26$ & 0.527 \\
6MWT & $333.43 \pm 86.58$ & $348.46 \pm 81.37$ & 0.542 \\
MET & $9.37 \pm 2.37$ & $8.83 \pm 3.55$ & 0.195 \\
Duration of exercise (minute) & $7.51 \pm 2.67$ & $6.65 \pm 2.02$ & 0.079 \\
Neutrophil/lymphocyte ratio & $2.01 \pm 1.22$ & $2.63 \pm 1.23$ & 0.343 \\
Platelet/lymphocyte ratio & $110.94 \pm 46.87$ & $120.97 \pm 26.95$ & 0.144 \\
Platelet count & $231.70 \pm 52.69$ & $256.82 \pm 65.62$ & 0.023 \\
Neutrophil count & $4.14 \pm 1.51$ & $5.49 \pm 2.40$ & 0.471 \\
Lymphocyte count & $2.32 \pm 0.81$ & $2.18 \pm 0.64$ & 0.241 \\
\hline Significance test between the two means was used & &
\end{tabular}

continuous variables were presented as mean \pm standard deviation while the data of categorical variables were given as $\mathrm{n}(\%)$. The differences between group means were compared using the significance test for the difference between two means for quantitative variables. The Pearson correlation coefficient was used to determine the relationships between quantitative variables. The $\mathrm{p}$ values lower than 0.05 were considered statistically significant. Statistical analyses were performed using SPSS software (IBM SPSS Statistics ver. 19, SPSS Inc., an IBM Co., Somers, NY).

\section{Results}

Of all participants, $49 \%(n=25)$ were female and $51 \%(n$ $=26$ ) were male. Twenty-three patients with coronary artery disease diagnosis $(45.1 \%)$ were included in a cardiac rehabilitation program while 14 patients $(27.5 \%)$ with asthma diagnosis and 14 patients $(27.5 \%)$ with COPD diagnosis were enrolled in a pulmonary rehabilitation program. No significant differences $(\mathrm{p}$ $>0.05$ ) were found between cardiac and pulmonary rehabilitation groups except for platelet count. The average age was $60.04 \pm 6.79$ years in the cardiac rehabilitation group and $54.07 \pm 11.08$ years in the pulmonary rehabilitation group. In the cardiac rehabilitation group, the mean 6MWT distance was $333.43 \pm 86.58$ meters and the mean MET value was $9.37 \pm 2.37$. The same values were $348.46 \pm 81.37$ meters and $8.83 \pm 3.55$ for the pulmonary rehabilitation group. Neutrophil/lymphocyte ratio was $2.01 \pm 1.22$ in the cardiac rehabilitation group and $2.63 \pm 1.23$ in the pulmonary rehabilitation group (Tab. 1).

The resting peak heart rate, Borg score, MET and duration of exercise were not correlated with other variables in the cardiac rehabilitation group. The 6MWT values were positively correlated with the exercise test peak heart rate $(\mathrm{p}<0.05)$. A significant and negative correlation was found between 6MWT and neutrophil/ lymphocyte ratio $(\mathrm{p}<0.05)$. In addition, the relationship between neutrophil/lymphocyte ratio and platelet/lymphocyte ratio was also significant $(\mathrm{p}<0.05)$ (Tab. 2).

In the pulmonary rehabilitation group, the resting peak heart rate was positively correlated with the exercise test peak heart rate and exercise test percent of peak heart rate $(p<0.05)$. The duration of exercise and MET increased along with the increase in 6MWT distance $(\mathrm{p}<0.05)$. The increase in MET was positively correlated with the exercise test peak heart rate, exercise

Tab. 2. Correlations between quantitative variables in the cardiac the rehabilitation group.

\begin{tabular}{|c|c|c|c|c|c|c|c|c|c|c|}
\hline & & & 1 & 2 & 3 & 4 & 5 & 6 & 7 & 8 \\
\hline 1 & Resting peak heart rate & $\mathrm{r}$ & 1 & .116 & .101 & .016 & .229 & -.359 & -.255 & -.216 \\
\hline 2 & Exercise test peak heart rate & $\mathrm{r}$ & .116 & 1 & $.868 *$ & -.395 & $.659 *$ & .221 & .104 & -.310 \\
\hline 3 & Exercise test peak heart rate $(\%)$ & $\mathrm{r}$ & .101 & $.868 *$ & 1 & -.304 & $.628 *$ & .348 & .043 & -.113 \\
\hline 4 & Borg score & $\mathrm{r}$ & .016 & -.395 & -.304 & 1 & -.037 & .206 & -.304 & -.038 \\
\hline 5 & 6MWT & $\mathrm{r}$ & .229 & $.659 *$ & $.628 *$ & -.037 & 1 & .290 & -.183 & $-.633 *$ \\
\hline 6 & MET & $\mathrm{r}$ & -.359 & .221 & .348 & .206 & .290 & 1 & .312 & -.059 \\
\hline 7 & Duration of exercise & $\mathrm{r}$ & -.255 & .104 & .043 & -.304 & -.183 & .312 & 1 & .410 \\
\hline 9 & Platelet/lymphocyte ratio & $\mathrm{r}$ & -.148 & -.385 & -.291 & .262 & -.385 & -.156 & .100 & $.598 *$ \\
\hline
\end{tabular}

Pearson correlation coefficient was used. * $\mathrm{p}<0.05$ 
Tab. 3. Correlations between quantitative variables in the pulmonary rehabilitation group.

\begin{tabular}{|c|c|c|c|c|c|c|c|c|c|c|}
\hline & & & 1 & 2 & 3 & 4 & 5 & 6 & 7 & 8 \\
\hline 1 & Resting peak heart rate & $\mathrm{r}$ & 1 & $.450 *$ & $.385 *$ & -.099 & -.171 & -.161 & .055 & -.076 \\
\hline 2 & Exercise test peak heart rate & $\mathrm{r}$ & $.450 *$ & 1 & $.691 *$ & -.362 & .236 & $.394 *$ & $.383^{*}$ & -.254 \\
\hline 3 & Exercise test peak heart rate $(\%)$ & $\mathrm{r}$ & $.385^{*}$ & $.691 *$ & 1 & -.371 & .314 & $.536^{*}$ & $.407 *$ & -.176 \\
\hline 4 & Borg score & r & -.099 & -.362 & -.371 & 1 & -.202 & -.212 & -.230 & -.064 \\
\hline 5 & 6MWT & $\mathrm{r}$ & -.171 & .236 & .314 & -.202 & 1 & $.495 *$ & $.475^{*}$ & -.175 \\
\hline 6 & MET & r & -.161 & $.394 *$ & $.536^{*}$ & -.212 & $.495 *$ & 1 & $.499 *$ & $-.463 *$ \\
\hline 7 & Duration of exercise & $\mathrm{r}$ & .055 & $.383^{*}$ & $.407 *$ & -.230 & $.475^{*}$ & $.499 *$ & 1 & $-.448 *$ \\
\hline 9 & Platelet/lymphocyte ratio & $\mathrm{r}$ & -.120 & .061 & -.109 & -.033 & -.199 & -.106 & .059 & .277 \\
\hline
\end{tabular}

Pearson correlation coefficient was used. $* \mathrm{p}<0.05$

test percent of peak heart rate, 6MWT and duration of exercise (p $<0.05$ ). It was observed that along with longer exercise time the exercise test peak heart rate, exercise test percent of peak heart rate, 6MWT and MET values increased $(\mathrm{p}<0.05)$. Higher neutrophil/lymphocyte ratios were negatively correlated with MET value and duration of exercise $(p<0.05)$. The platelet/lymphocyte ratio and Borg score were not significantly correlated with other variables (Tab. 3).

\section{Discussion}

Leucocyte activation and migration of cells from blood to the inflammation area could play a significant role in decreased exercise capacity $(15,16)$. In addition, in previous studies it was suggested that proinflammatory cytokines (tumor necrosis factor alpha and interleukin-1) could modulate cardiac functions, peripheric vascular functions and exercise performances through mechanisms such as production of free oxygen radicals and apoptosis (15-18). In the present study, increased NLR values were correlated with lower exercise capacity. In a study evaluating the association between preoperative exercise capacity and inflammatory markers, the impaired cardiovascular performance measured by cardiopulmonary exercise test was correlated with leucocyte changes (19). In the same study, decreased CD14 monocyte expression and lymphopenia were associated with impaired cardiorespiratory capacity.

As NLR increased, the 6MWT distance decreased in the patient group with atherosclerotic cardiovascular disease for which cardiac rehabilitation program was planned. Inflammation plays a role in the onset, progression and destabilization of atherosclerotic plaque. Systemic inflammation is known to be associated with inflammation in vessel walls (20). Lymphocytes and monocytes play roles in early stages of plaque while neutrophils are involved in acute disruption of plaque and thrombotic occlusion $(20,21)$. In individuals with atherosclerotic cardiovascular disease, the NLR increase could affect physical performance. Immune dysregulation is known as an important characteristic of poor aerobic capacity (22). Platelets are also thought to play a major role in the pathogenesis of atherosclerosis. Platelet/lymphocyte ratio (PLR) has been shown to be associated with all-cause mortality in acute myocardial infarction patients (23). Systemic infection lowers the lifetime of erythrocytes and platelets and results in membrane deformity. The high red cell distribution width and mean platelet volume were reported to be associated with elevated inflammation in various diseases (24-26). In the present study, NLR was associated with PLR, but no relationship was found between PLR and exercise capacity.

In the present study, the NLR increase tended to have an association, though not significant, with MET decreases in patients for whom cardiac rehabilitation was planned. Similarly, in another study dealing with individuals with idiopathic dilated cardiomyopathy diagnosis, the elevated NLR was associated with poor functional capacity (27). Functional capacity, expressed as MET based on treadmill test, is one of the major prognostic factors in individuals with heart failure. The NLR value with a threshold level of 2.26 predicts a poor functional capacity with the sensitivity of $83 \%$ and specificity of $69 \%$ (27). In a study by Cakici et al, functional capacity $(3.2 \pm 2.05$ MET and $6.1 \pm 2.04$ MET; $p$ $<0.001)$ and ejection fraction $(31.5 \% \pm 7.64$ and $34.8 \% \pm 6.82$; $\mathrm{p}=0.028$ ) were lower in patients with heart failure whose NLR was over 3. NLR threshold level of 2.74 had a $79.4 \%$ sensitivity and $80 \%$ specificity in predicting the low functional capacity. Therefore, NLR was proposed as a useful tool to evaluate exercise performance of patients with heart failure (28). Similarly, NLR could also be used to predict functional capacity of patients before cardiac rehabilitation program.

The present study showed that MET decreased along with increasing NLR in a pulmonary rehabilitation program. Frutate et al (6) evaluated the association between NLR and clinical parameters of stable period in 141 COPD patients. They also studied a potential change during exacerbations of disease in 49 patients. NLR was significantly associated with forced expiratory volume in the first second (FEV 1), BODE index and 6MWT. Similar to the present study, NLR was associated with exercise capacity and was also reported to be a possible predictor of COPD severity and clinical performance (6). Though not significant, NLR had a negative correlation with 6MWT distance in the present study. Lee et al (12) also reported a negative relationship between NLR and 6MWT test distances in COPD patients. In a similar study dealing with the association between NLR and clinical parameters in COPD patients, NLR was significantly associated with BODE index and 6MWT distance (29). Thus, the NLR value measured before the pulmonary rehabilitation program could be used to estimate the exercise capacity of the patients. 


\section{Study limitations}

The present study is the first to evaluate the association between NLR value and exercise capacity in individuals for whom cardiopulmonary rehabilitation program is planned. Nevertheless, the study has some limitations. Some associations may not have reached the significance level because of a relatively low number of patients and single-center nature of the study. Due to the lack of sufficient equipment for the exercise test, the cardiopulmonary exercise test was not used and therefore the association between NLR and peak oxygen capacity was not established. Because of the sectional structure of the study, the relationship between NLR and performance of patients during the rehabilitation program was not studied. Therefore, larger cohort studies are needed to evaluate the association between NLR and functional capacity changes of the patients at the end of the rehabilitation program.

\section{Conclusion}

A previous study reported that measuring the starting NLR value could be used to distinguish the patients who could benefit from cardiac resynchronization therapy (30). Another area in which NLR value might be useful are cardiopulmonary rehabilitation programs. As an easily accessible parameter for individuals who are to be enrolled in cardiopulmonary rehabilitation program, NLR could be used to predict the exercise capacity and functional capacity, and to estimate MET value and distance that patients could cover in 6MWT test.

- Neutrophil/lymphocyte ratio has recently been used as a potential marker to determine inflammation in cardiac and noncardiac diseases.

- Cardiopulmonary rehabilitation programs are considered a part of comprehensive care for the patients with cardiopulmonary disease.

- Neutrophil/lymphocyte ratio could be used as a predictor to evaluate the exercise capacity in patients who are to be enrolled in a cardiopulmonary rehabilitation program.

\section{References}

1. Smith S, Chaudhary K, Blackstock F. Concordant Evidence-Based Interventions in Cardiac and Pulmonary Rehabilitation Guidelines. J Cardiopulmon Rehab Prevent 2019; 39: 9-18.

2. Sarikaya $\mathbf{S}$, Tur BS, Kurtaiş $\mathbf{Y}$ et al. The awareness of physicians and allied health professionals about cardiopulmonary rehabilitation: A cross-sectional survey study. Turk J Phys Med Rehab 2014; 60(1): 19-24.

3. Duffy BK, Gurm HS, Rajagopal V, Gupta R, Ellis SG, Bhatt DL. Usefulness of an elevated neutrophil to lymphocyte ratio in predicting long term mortality after percutaneous coronary intervention. Amer J Cardiol 2006; 97: 993-996.

4. Pascual-González Y, López-Sánchez M, Dorca J, Santos S. Defining the role of neutrophil-to-lymphocyte ratio in COPD: a systematic literature review. Internat J COPD 2018; 13: 3651-3662.
5. Zahorec R. Ratio of neutrophil to lymphocyte counts: rapid and simple parameter of systemic inflammation and stress in critically ill. Bratisl Lek Listy 2001; 102: 5-14.

6. Furutate R, Ishii T, Motegi T, Hattori K, Kusunoki Y, Gemma A, Kida K. The neutrophil to lymphocyte ratio is related to disease severity and exacerbation in patients with chronic obstructive pulmonary disease. Intern Med 2016; 55: 223-229.

7. Balady GJ, Williams MA, Ades PA et al. Core components of cardiac rehabilitation/secondary prevention programs: 2007 update: A scientific statement from the American heart association exercise, cardiac rehabilitation, and prevention committee, the council on clinical cardiology; the councils on cardiovascular nursing, epidemiology and prevention, and nutrition, physical activity, and metabolism; and the American association of cardiovascular and pulmonary rehabilitation. Circulation 2007; 115 (20): 2675-2682.

8. Piepoli MF, Corra 'U, Benzer W et al. Secondary prevention through cardiac rehabilitation: from knowledge to implementation. A position paper from the Cardiac Rehabilitation Section of the European Association of Cardiovascular Prevention and Rehabilitation European. J Cardiovasc Prevent Rehab 2010; 17: 1-17.

9. Mancini DM, Eisen H, Kussmaul W, Mull R, Edmunds LH, Wilson JR. Value of peak exercise oxygen consumption for optimal timing of cardiac transplantation in ambulatory patients with heart failure. Circulation 1991; 83: 778-786.

10. Fleg JL, Pina IL, Balady GJ et al. Assessment of functional capacity in clinical and research applications: An advisory from the Committee on Exercise, Rehabilitation, and Prevention, Council on Clinical Cardiology. American Heart Association. Circulation 2000; 102: 1591-1597.

11. Enright PL, Sherrill DL. Reference equations for the six-minute walk in healthy adults. Amer J Respir Crit Care Med 1998; 158: 1384-1387.

12. Lee H, Um S-J, Kim YS et al. Association of the NeutrophiltoLymphocyte Ratio with Lung Function and Exacerbations in Patients with Chronic Obstructive Pulmonary Disease. PLoS ONE 2016; 11 (6): e0156511.

13. American Thoracic Society statement: guidelines for the six-minute walk test. Amer J Respir Crit Care Med 2002; 166: 111e7.

14. Bruce R, Blackmon J, Jones J, Strait G. Exercising testing in adult normal subjects and cardiac patients. Pediatrics 1963; 32 (4): 742-756.

15. Adamopoulos $\mathrm{S}$, Parissis J, Kroupis $\mathrm{C}$ et al. Physical training reduces peripheral markers of inflammation in patients with chronic heart failure. Eur Heart J 2001; 22: 791-797.

16. Pinsky DJ, Cai B, Yang X, Rodriguez C, Sciacca RR, Cannon PJ. The lethal effects of cytokine-induced nitric oxide on cardiac myocytes are blocked by nitric oxide synthase antagonism or transforming growth factor beta. J Clin Invest 1995; 95: 677-685.

17. Mann DL, Young JB. Basic mechanisms in congestive heart failure. Recognizing the role of proinflammatory cytokines. Chest 1994; 105: 897-904.

18. Devaux B, Scholz D, Hirche A, Klövekorn WP, Schaper J. Upregulation of cell adhesion molecules and the presence of low grade inflammation in human chronic heart failure. Eur Heart J 1997; 18 : 470-479.

19. Sultan P, Edwards MR, Arroyo AG et al. Cardiopulmonary Exercise Capacity and Preoperative Markers of Inflammation. Hindawi Publishing Corporation Mediators of Inflammation 2014; 727451: 8. 


\section{6-210}

20. Libby P. Inflammation in atherosclerosis. Arterioscler Thromb Vasc Biol 2012; 32: 2045-2051.

21. Yayan J. Emerging families of biomarkers for coronary artery disease: Inflammatory mediators. Vascular Health and Risk Management 2013; 9: 435-456.

22. Topkara VK, Evans S, Zhang W, Epelman S, Staloch L, Berger PM, Mann DL. Therapeutic targeting of innate immunity in the failing heart. J Mol Cell Cardiol 2011; 51(4): 594-599.

23. Azab B, Shah N, Akerman M, McGinn JT. Value of platelet/lymphocyte ratio as a predictor of all-cause mortality after non-ST-elevation myocardial infarction. J Thromb Thrombolysis 2012; 34: 326-334.

24. Xanthopoulos A, Giamouzis G, Melidonis A et al. Red blood cell distribution width as a prognostic marker in patients with heart failure and diabetes mellitus. Cardiovasc Diabetol 2017; 16: 81.

25. Tasoglu Ö, Sahin A, Karatas G, Koyuncu E, Tasoglu I, Tecimel O, Özgirgin N. Blood mean platelet volume and platelet lymphocyte ratio as new predictors of hip osteoarthritis severity. Medicine (Baltimore) 2017; 96: e6073.
26. Tanrikulu CS, Tanrikulu Y, Sabuncuoglu MZ, Karamercan MA, Akkapulu N, Coşku F. Mean platelet volume and red cell distribution width as a diagnostic marker in acute appendicitis. Iranian Red Crescent Med J 2014; 16: e10211.

27. Yildiz A, Yüksel M, Oylumlu M, Polat N, Akil MA, Acet H. The association between the neutrophil/lymphocyte ratio and functional capacity in patients with idiopathic dilated cardiomyopathy. Anatolian $\mathrm{J}$ of Cardiology 2015; 15: 13-17.

28. Çakici M, Çetin M, Doğan A et al. Neutrophil to lymphocyte ratio predicts poor functional capacity in patients with heart failure. Arch Turk Soc Cardiol 2014; 42(7): 612-620.

29. Lee SJ, Lee HR, Lee TW et al. Usefulness of neutrophil to lymphocyte ratio in patients with chronic obstructive pulmonary disease: a prospective observational study. Korean J Intern Med 2016; 31 (5): 891-898.

30. Agacdiken A, Celikyurt U, Sahin T, Karauzum K, Vural A, Ural D. Neutrophil-to-lymphocyte ratio predicts response to cardiac resynchronization therapy. Med Sci Monitor 2013; 19: 373-377.

Received October 1, 2019. Accepted December 18, 2019. 